\title{
Elaboração de Atividades Profissionais Confiáveis (APCs) em ginecologia e obstetrícia para a graduação médica
}

\section{Creation of Entrustable Professional Activities (EPAs) in obstetrics and gynecology for medical undergraduate students}

Andrea Mora De Marco Novellino' (1) andreanovellino@hotmail.com Izabel Cristina Meister Martins Coelho' (1) izamcoelho@gmail.com

\section{RESUMO}

Introdução: Este artigo traz "Atividades Profissionais Confiáveis" (APCs) na área de ginecologia e obstetrícia elaboradas para a graduação médica a fim de sustentar o processo de ensino-aprendizagem.

Objetivo: O objetivo é que o egresso seja competente para atender aos requisitos mínimos necessários ao estudante nesta área.

Método: Neste estudo, as APCs foram criadas com base num molde internacional já utilizado e validadas pela técnica Delphi. A estruturação das APCs foi baseada na literatura internacional: "Principais atividades profissionais confiáveis (APCs) para acesso à Residência Médica: Guia para Elaboradores de Currículos" da Associação Americana de Faculdades Médicas (AAMC). O conteúdo dos temas para elaboração de cada APC foi baseado na publicação "Pilares da Obstetrícia e Ginecologia" oriundo da ação conjunta do Conselho de Acreditação de Educação Médica na Graduação (ACGME), Colégio Americano de Ginecologia e Obstetrícia (ACOG) e pelo Conselho Americano de Ginecologia e Obstetrícia (ABOG).

Resultados: Foram elaboradas treze APCs com temas para o ensino da referida área durante a graduação. Para cada APC, uma lista de verificação foi elaborada de forma a orientar o avaliador e o estudante quanto à execução das tarefas definidas nas APCs.

Conclusão: As APCs com as listas de verificação trazem uma proposta inovadora no ensino da ginecologia e obstetrícia na graduação médica, uma vez que facilitam e operacionalizam o aprendizado, o desenvolvimento e a observação da aquisição dos conhecimentos, habilidades e atitudes exigidos na execução de cada tema na área, auxiliando, assim, a formação de um profissional competente. Ao final do curso de medicina, a capacidade de realização de todas as APCs identifica um egresso competente em ginecologia e obstetrícia naquilo que concerne ao médico generalista.

Palavras-chave: Atividades Profissionais Confiáveis; Educação Médica; Competência Clínica.

\section{ABSTRACT}

Introduction: This article features "Entrustable Professional Activities (EPAs)" in the Obstetrics and Gynecology field designed formedical undergraduates, aiming to support the teaching/learning process.

Objective: The aim is for the graduate to be competent to meet the minimum requirements necessary for the student in this field.

Method: In this study, EPAs were created based on an international template already in use and validated using the Delphi technique. The structuring of the EPAs was based on the international literature: "Core Entrustable Professional Activities for Entering Residency: Curriculum Developer's Guide" from the Association of American Medical Colleges (AAMC). The content for the topics of each EPA was based on the publication "The Obstetrics and Gynecology Milestone Project" from the joint action of the Accreditation Council for Graduate Medical Education (ACGME), the American College of Obstetrics and Gynecology (ACOG) and the American Board of Obstetrics and Gynecology (ABOG).

Results: Thirteen EPAs were created with topics for teaching in that field during undergraduate school. A checklist was created for each EPA, aiming to guide the evaluator and the student regarding the performance of the tasks defined in the EPAs.

Conclusion: The EPAs with checklists bring an innovative proposal for Obstetrics and Gynecology teaching in medical graduation since they facilitate and operationalize the learning, the development, and the observation of the acquisition of knowledge, skills and attitudes required for the performance of each topic in the area, thus helping the training of a competent professional. At the end of the medical course, the ability to perform all EPAs identifies a competent graduate in Gynecology and Obstetrics regarding the general practitioner.

Keywords: Entrustable Professional Activities; Medical Education; Clinical Competence.

${ }^{1}$ Faculdades Pequeno Príncipe, Curitiba, Paraná, Brasil.

Editora-chefe: Rosiane Viana Zuza Diniz.

Editor associado: Pedro Tadao Hamamoto Filho.

Recebido em 11/10/20; Aceito em 30/07/21.

Avaliado pelo processo de double blind review. 


\section{INTRODUÇÃO}

A necessidade de assumir a responsabilidade com a saúde da população e a proteção dos cidadãos contra a prática profissional médica incompetente levou ao conceito de competência e à identificação dos componentes do currículo médico'. Ao recolocar a prática profissional como foco, impõese às escolas repensar seus currículos e processos avaliativos, reorientando o planejamento educacional a partir do perfil de competência desejável para seus egressos ${ }^{2}$. Qualidades mais difíceis de avaliar - mas fundamentais, como ética, profissionalismo, comunicação e liderança - também devem ser buscadas e avaliadas durante o desenvolvimento profissional. Não basta adquirir conhecimentos e habilidades ${ }^{3}$; é preciso desenvolver ações para atingir os objetivos finais, aplicando os conhecimentos e habilidades que foram adquiridos.

Para uma educação médica que seja abrangente e integradora, há a necessidade de se definir as competências esperadas para o profissional que se deseja formar e averiguar se essas competências foram alcançadas ao final da graduação médica. Mas como saber se o estudante realmente as desenvolveu na graduação? Neste contexto, apresentamos as Atividades Profissionais Confiáveis (APCs) em ginecologia e obstetrícia com suas respectivas listas de verificação para auxiliar o processo de ensino-aprendizagem e a avaliação das competências adquiridas durante a graduação médica.

As APCs são unidades de prática profissional definidas como tarefas ou responsabilidades que os estudantes em desenvolvimento têm que realizar sem supervisão direta, uma vez que eles tenham suficientemente alcançado uma competência específica. As APCs são executáveis, observáveis e mensuráveis nos seus processos e resultados; portanto, adequadas para tomada de decisões confiáveis. As APCs são um meio para traduzir as competências na prática clínica. Competências são descritores de médicos, APCs são descritores de trabalho ${ }^{4}$.

O conceito foi desenvolvido para operacionalizar a educação baseada em competências na pós-graduação médica, no modelo de residência médica ${ }^{5,6}$, mas é agora amplamente aplicado na educação de profissionais de saúde ${ }^{7,8}$. Nessa perspectiva, um projeto que alinhe os objetivos que devem ser alcançados ao final da graduação com o que é esperado do egresso no primeiro dia da residência médica — ou de atuação no mercado de trabalho - pode contribuir para a formação integral do médico.

As APCs se operacionalizam como atividades profissionais essenciais que devem ser confiadas a um profissional para realizá-las. Cada APC é uma síntese de vários domínios de competência (por exemplo, conhecimento médico, habilidades de comunicação e profissionalismo) e requer a integração de conhecimentos, habilidades e atitudes ${ }^{9}$. São unidades da prática profissional que constituem o fazer diário dos clínicos ${ }^{1,5}$ e constituem uma lista de tarefas a serem realizadas em determinado período de tempo no cuidado ao paciente.

A indagação se o estudante da área da saúde adquiriu competência para a profissão ao final do curso é papel de cada professor, cabendo a esse adquirir uma visão longitudinal dos objetivos de ensino e administrar a progressão da aprendizagem dos estudantes ${ }^{10}$.

APCs e competências não são mutuamente exclusivas; pelo contrário, elas exigem integração de competências, e as competências são mais bem avaliadas no contexto do desempenho, da prática, como pode ser fornecido pela estrutura de uma $A P C^{11}$. Nesse sentido, são necessárias ferramentas capazes de visualizar os pontos fortes no processo de formação acadêmica, bem como proporcionar intervenções nos pontos fracos, objetivando a formação de médicos competentes na área da saúde, com destaque para a atenção básica em saúde. A execução da APC pelo estudante sob supervisão permite observar os pontos frágeis na formação médica, corrigir esses pontos antes do acesso à residência médica ou ao mercado de trabalho e proporcionar ao próprio estudante a visualização do que precisa ser aprimorado no seu aprendizado.

Esta é uma proposta inovadora para o ensino da Ginecologia e Obstetrícia (G.O.) durante a graduação médica que tem como objetivo principal auxiliar o estudante a alcançar a competência desejada na área e, como objetivo secundário, auxiliar o docente a avaliar o estudante quanto à aquisição de determinada competência.

\section{METODOLOGIA}

O presente estudo foi aprovado pelo Comitê de Ética e Pesquisa sob o parecer número 2.786.783.

A metodologia utilizada na elaboração das APCs é internacionalmente aceita. Trata-se de uma proposta inicial, baseada em modelos internacionais, que serviram de referências norteadoras na elaboração das APCs: o "Guia para Elaboradores de Currículos: Principais Atividades Profissionais Confiáveis (APCs) para acesso à Residência Médica"11 e o trabalho desenvolvido em conjunto pelo Conselho de Acreditação de Educação Médica na Graduação (ACGME), Colégio Americano de Obstetrícia e Ginecologia (ACOG) e pelo Conselho Americano de Obstetrícia e Ginecologia (ABOG), intitulado “Projeto Pilares da Obstetrícia e Ginecologia" (The Obstetrics and Gynecology Milestone Project) ${ }^{12}$.

Para cada APC, foi elaborada uma lista de verificação para avaliar a progressão nas tarefas. A lista de verificação foi baseada na descrição das funções da atividade que o estudante deve desempenhar na execução da APC correlata. Foram construídas treze APCs e treze listas de verificação correspondentes a cada uma das APCs. 
Essa proposta passou por um processo de validação de conteúdo denominado técnica Delphi, que ocorreu de forma totalmente online durante um período aproximado de quatro meses para a coleta dos dados e envolveu peritos na área. Foram selecionados 32 peritos de Instituições de Ensino Superior públicas e privadas. Os critérios de elegibilidade para inclusão de peritos foram: ser médico especialista em ginecologia e obstetrícia e professor universitário nesta mesma área na graduação em medicina. O tempo de atuação de cada painelista sendo especialista e professor variou entre seis e doze anos. Eles foram abordados individualmente por meio de correio eletrônico e convidados a responder a um questionário avaliando as APCs, conjuntamente com suas respectivas listas de verificação, e emitir sugestões sobre o conteúdo.

O questionário para avaliação dos peritos foi elaborado da seguinte forma: na primeira rodada foram apresentadas as treze APCs elaboradas juntamente com suas listas de verificação correspondentes. Para cada APC foram feitas quatro perguntas descritas abaixo:

- A APC está descrita de forma clara, inequívoca e objetiva permitindo que o professor identifique facilmente o que deve ser executado?

- A APC é relevante no ensino da G.O. na graduação?

- A APC é pertinente ao ensino da G.O. para a graduação?

A concordância nas respostas foi avaliada de acordo com as respostas em uma escala Likert para cada pergunta, graduada de 1 (discordo totalmente) a 5 (concordo totalmente). Por último, foi feita a quarta pergunta para resposta aberta em texto que o painelista poderia ou não responder de acordo com sua avaliação da APC:

- A partir de sua experiência, você gostaria de acrescentar alguma sugestão, comentário ou mudança no texto?

Cada lista de verificação foi apresentada no questionário logo abaixo da sua respectiva APC e feitas exatamente as mesmas quatro perguntas.

Retornaram 20 respostas na primeira rodada. As APCs propostas receberam elogios quanto à elaboração e ao conteúdo que foi avaliado referente à clareza, relevância e pertinência. Houve concordância de $100 \%$ quanto à relevância e pertinência, e $85 \%$ quanto à clareza. Neste item, algumas sugestões foram encaminhadas por escrito tais como: "alterar a ordem dos itens" e "pequena alteração no título", que foram aceitas e modificadas no texto. Outra sugestão foi a elaboração de tema para construção de mais APCs, o que, neste caso específico, não foi incluído no presente trabalho, pois a sugestão não cabia para ensino na graduação; tratava-se de tema que concerne ao especialista em ginecologia e obstetrícia durante a residência médica; portanto, não se adequando à proposta em questão que objetiva o uso da APC na graduação. Desta forma, após incorporação das sugestões, uma segunda rodada foi realizada entre os 20 respondentes dos quais recebemos 18 respostas com concordância de todos os envolvidos. A tabela 1 traz a participação dos respondentes em cada rodada.

A validação de conteúdo da APC aponta se ela é realmente parte do trabalho diário de acordo com sua definição e se é adequada para seu propósito. A validação de um conjunto de APCs também visa abranger as atividades de uma profissão. Isso pode ser feito com parecer de peritos. O parecer do perito não só garante a qualidade do conjunto de APCs, mas também informa e envolve professores que poderão trabalhar com essas APCs no futuro ${ }^{13}$ (Figura 1).

\section{Elaboração das APCs}

Foram construídas treze APCs próprias para o ensino da ginecologia e obstetrícia na graduação médica baseadas no Guia para Elaboradores de Currículos: Principais Atividades

Tabela 1. Participantes em cada rodada e duração.

\begin{tabular}{cccc}
\hline Rodadas & Duração & $\begin{array}{c}\text { Participantes } \\
\text { (Receberam } \\
\text { questionário) }\end{array}$ & $\begin{array}{c}\text { Participantes } \\
\text { (Respondentes) }\end{array}$ \\
\hline $1^{\text {a Rodada }}$ & 63 dias & 32 & 20 \\
\hline $2^{\text {a }}$ Rodada & 50 dias & 20 & 18 \\
\hline
\end{tabular}

Figura 1. Passos envolvidos na elaboração e validação das APCs.

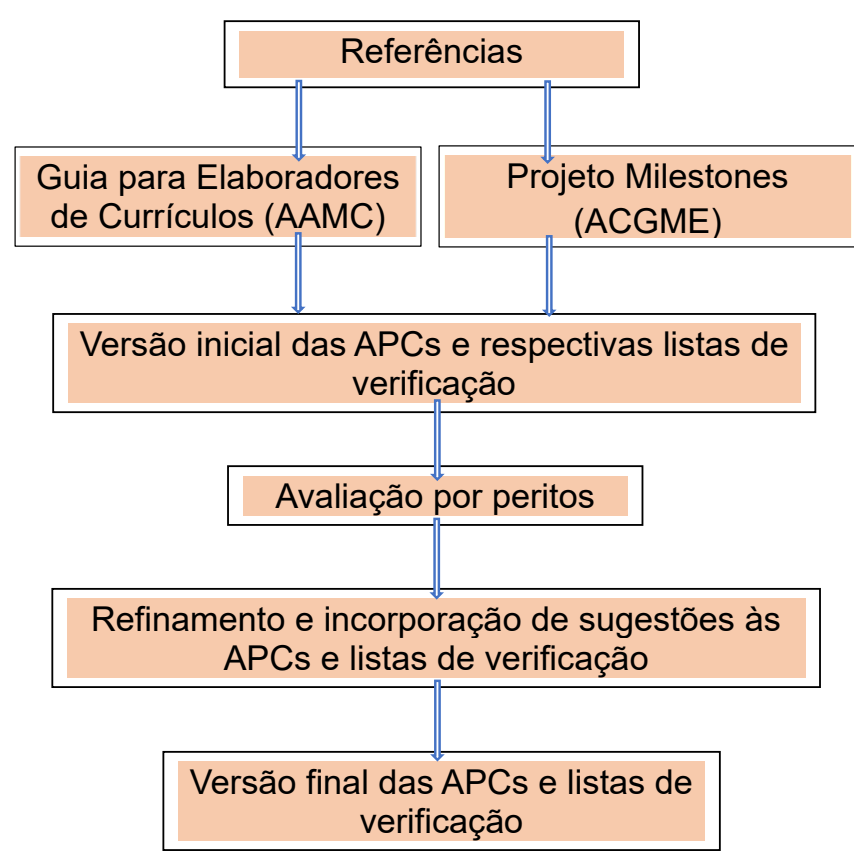


Profissionais Confiáveis (APCs) para acesso à Residência Médica ${ }^{11}$, e no Projeto Pilares da Ginecologia e Obstetrícia (Milestones Project) ${ }^{12}$ oriundo da ação conjunta do Conselho de Acreditação de Educação Médica na Graduação (ACGME), Colégio Americano de Obstetrícia e Ginecologia (ACOG) e pelo Conselho Americano de Obstetrícia e Ginecologia (ABOG).

Os Pilares foram concebidos para uso na avaliação de médicos residentes, no âmbito da sua participação nos programas de residência médica. Esses fornecem uma estrutura para a avaliação do desenvolvimento do médico residente em atributos-chave dos elementos de competência médica em uma especialidade ou subespecialidade. Porém coube aqui sua utilização no sentido visionário daquilo que se espera do egresso no seu primeiro dia de formado correlacionando-se ao nível 1 dos Pilares. Esses são organizados em níveis numerados, e o seguimento do nível 1 para o nível 5 é sinônimo de passar de iniciante a especialista.

A ideia inicial deste estudo era adequar as APCs já existentes internacionalmente no material da $\mathrm{AAMC}^{11}$ para utilização no ensino da ginecologia e obstetrícia na graduação. Porém esta foi uma limitação encontrada uma vez que não contemplaria várias práticas que são próprias da especialidade. Sendo assim optamos por elaborar APCs específicas com temas em ginecologia e obstetrícia.

As APCs contemplam as funções que o estudante deve desempenhar sobre determinado tema de forma ideal até que possa realizar a tarefa sem supervisão direta ao final da graduação. A estrutura de cada APC conta com um cabeçalho com o título do tema que é abordado na APC, com espaço para o nome do estudante, do avaliador e a data da realização. $O$ estudante pode repetir a execução da APC em diferentes datas ao longo do curso, com o mesmo ou outro avaliador, e assim ter condições de analisar sua progressão na execução da atividade. Constam ainda da estrutura de cada APC: a descrição da atividade a ser realizada com indicação de qual conhecimento o estudante tem que ter previamente; as funções nessa atividade, indicando as habilidades que ele deve desenvolver e realizar na execução da APC; os domínios de competência relevantes nessa atividade; o local para o avaliador marcar como o estudante deve ainda atuar na execução daquela APC em relação à supervisão direta ou indireta. Os domínios de competência relevantes no desenvolvimento de cada APC estão descritos em cada atividade como "Domínios de competência relevantes nesta atividade" e foram extraídas do Guia para Elaboradores de Currículos: Principais Atividades Profissionais Confiáveis (APCs) para acesso à Residência Médica ${ }^{11}$.

No modelo sugerido pela Associação Americana de Faculdades Médicas (AAMC), que elaborou as APCs gerais, não consta lista de verificação associada a cada APC, sendo a elaboração dessa um dos objetivos desta pesquisa.

Cumpridos - ou não - todos os itens da lista de verificação, o avaliador poderá considerar de que forma o estudante pode atuar no desempenho da tarefa proposta pela APC em questão, sendo assim descritos:

- "Deve somente atuar sob supervisão direta"

- "Pode atuar sob supervisão indireta ou a distância"

- "Poderia atuar sem supervisão" - este item identifica a competência alcançada, porém a atuação sem supervisão não é realizada na graduação por questões legais.

O estudante se propõe a desempenhar as tarefas descritas na APC a fim de alcançar os domínios relevantes e desenvolver as habilidades específicas solicitadas na atividade em questão até que alcance o nível "Pode atuar sem supervisão", que é o esperado para o egresso do curso de medicina. Para visualização do seu progresso no desempenho da atividade proposta na APC, o avaliador assinala uma das opções no campo "Progressão nesta APC".

Para elaboração do conteúdo em ginecologia e obstetrícia de cada APC, os assuntos foram adaptados a partir do que consta no nível 1 de cada tema do "Projeto Pilares da Obstetrícia e Ginecologia" (Milestone Project). Esse nível 1 foi considerado o "marco" a ser atingido pelo estudante. Nesse ponto, o avaliador pode considerá-lo apto ou "confiável" para realizar determinada tarefa.

\section{RESULTADOS}

Os temas das treze APCs elaboradas foram:

APC 1: Realizar a anamnese (com foco em G.O.).

APC 2: Realizar o exame físico da mulher não gestante (Ginecológico).

APC 3: Realizar o exame físico da mulher gestante (Obstétrico).

APC 4: Atendimento a pacientes no período intraparto.

APC 5: Realizar o exame físico da puérpera.

APC 6: Realizar a coleta de exame Papanicolaou.

APC 7: Interpretar exames laboratoriais e de imagem comuns de diagnóstico e rastreio em G.O.

APC 8: Elaborar diagnóstico diferencial e hipótese diagnóstica.

APC 9: Princípios da cirurgia ginecológica e atendimento perioperatório.

APC 10: Abordagem à dor abdominal/pélvica (aguda e crônica) e à massa pélvica.

APC 11: Abordagem ao sangramento uterino anormal (agudo e crônico). 
APC 12: Abordagem ao sangramento de primeiro trimestre.

APC 13: Planejamento familiar e investigação do casal infértil.

O objetivo de cada APC é que o estudante desenvolva as competências necessárias para desempenhar a determinada tarefa que faz parte da vida profissional diária em ginecologia e obstetrícia, e seja capaz de realizá-la após a graduação médica. Os objetivos a serem atingidos em cada APC ficam explícitos na lista de verificação que avalia se o estudante foi capaz de desempenhar as tarefas propostas que culminarão com a realização completa da APC e consequentemente é apontado como apto para realizar o que foi proposto.

Abaixo seguem três exemplos das APCs propostas em ginecologia e obstetrícia com suas respectivas listas de verificação (LV), que podem ser aplicadas parcialmente desde o início do curso e reaplicados com maior complexidade até o final do curso e em egressos (Quadros 1, 2 e 3):

\section{Quadro 1. APC 3 - REALIZAR O EXAME FÍSICO DA MULHER GESTANTE (Obstétrico).}

APC 3

Estudante:

O estudante deve ser capaz de realizar - sem supervisão - um exame físico completo de forma organizada e sequencial, respeitando a paciente. O exame físico deve ser adaptado à situação clínica (gestante) e ao encontro específico da paciente (rotina de pré-natal ou urgência). A coleta de dados e a atividade de interação com a paciente servem de base para o trabalho clínico. Os estudantes precisam integrar os fundamentos científicos da medicina com habilidades de raciocínio clínico para orientar seu exame e sua coleta de informações.

Descrição da atividade

* Executar um exame físico completo e preciso da gestante na sequência lógica e fluida.

* Realizar um exame físico clinicamente relevante e focado pertinente à configuração e propósito da visita da paciente (consulta obstétrica).

Funções nesta atividade $\quad \begin{aligned} & \text { *Identificar, descrever e docum } \\ & \text { clareza no prontuário médico. }\end{aligned}$

*Demonstrar técnicas de exame centradas na paciente que refletem o respeito pela privacidade, conforto e segurança da paciente (por exemplo, explicando as manobras de exame físico, informando a paciente o que está fazendo em cada etapa, mantendo-a coberta durante o exame). *Demonstrar conhecimento e realizar os passos do exame físico completo da gestante.

*Cuidados com a paciente

* Conhecimento para a prática

*Habilidades interpessoais e de comunicação

*Profissionalismo

Domínios de competência

relevantes nesta atividade

( ) Deve somente atuar sob supervisão direta

Progressão nesta APC
() Pode atuar sob supervisão indireta ou à distância

( ) Pode atuar sem supervisão (nível 1 Milestones)

\section{LV - APC 3: realizar o exame físico da mulher gestante (obstétrico)}

Quando o estudante realiza o exame físico completo de uma mulher que vem à consulta de obstetrícia realizar a rotina do pré-natal ou por uma queixa específica:

- É capaz de executar um exame físico completo e preciso da mulher na sequência lógica e fluida? (Segue o exame no eixo axial, de cima para baixo, examinando cabeça, pescoço, membros superiores, tronco com ausculta pulmonar e cardiológica, mamas, abdômen gravídico, virilhas, membros inferiores, genitália externa e interna.)

- Realiza um exame físico clinicamente relevante e focado pertinente à configuração e propósito da visita da paciente? (Consulta obstétrica.)

- É capaz de comunicar-se eficazmente com a paciente, com as famílias e com o público, conforme apropriado, em uma ampla gama de origens socioeconômicas e culturais?

- Identifica, descreve e documenta descobertas anormais do exame físico, registrando com clareza no prontuário médico?

- Demonstra técnicas de exame centradas na paciente que refletem o respeito pela privacidade, conforto e segurança da paciente? (Explicando as manobras de exame físico, informando a paciente o que está fazendo em cada etapa, mantendo-a coberta durante o exame.)

- Demonstra conhecimento e realiza os passos do exame físico obstétrico básico? (Medida da altura uterina, ausculta de batimento cardiofetal, manobras de Leopold, toque vaginal.) 


\section{Quadro 2. APC 4 - ATENDIMENTO A PACIENTES NO PERÍODO INTRAPARTO.}

APC 4

Estudante:

O estudante deve demonstrar conhecimento básico de atendimento obstétrico intraparto de rotina e sem complicações, incluindo conduta de trabalho de parto normal. Fornecer atendimento obstétrico intraparto para mulheres com gestações sem complicações (identificar posição fetal, monitorar frequência cardíaca fetal e dinâmica uterina). Diferencia entre o trabalho de parto normal e anormal. Reconhece complicações intraparto (corioamnionite, distócia de ombro).

Descrição da atividade

Funções nesta atividade

*Identificar o início do traballho de parto.

* Conduzir o trabalho de parto normal fazendo ausculta do batimento cardiofetal, identificando posição fetal, avaliando a dinâmica uterina e a dilatação cervical através do toque vaginal.

* Preencher corretamente o partograma e interpretá-lo.

* Indicar corretamente e interpretar a cardiotocografia.

*Diferenciar o parto eutócico do parto distócico.

* Realizar o parto eutócico.

* Fornecer atendimento ao puerpério imediato.

*Reconhecer situação de urgência/emergência sendo capaz de comunicar-se com a equipe para solicitar apoio.

*Cuidados com a paciente

* Conhecimento para a prática

*Habilidades interpessoais e de comunicação

Domínios de competência relevantes nesta atividade

Colaboração interprofissional

*Aprendizagem e melhoria baseada em prática

*Profissionalismo

*Prática baseada em sistemas

Progressão nesta APC

() Deve somente atuar sob supervisão direta

() Pode atuar sob supervisão indireta ou a distância

( ) Pode atuar sem supervisão (nível 1 Milestones)

\section{LV-APC 4: atendimento a pacientes no período intraparto}

Quando o estudante está diante de um trabalho de parto:

- Identifica o início do trabalho de parto?(Sabe diferenciar trabalho de parto de falso trabalho de parto.)

- Conduz o trabalho de parto normal eutócico fazendo ausculta do batimento cardiofetal, identificando posição fetal, avaliando a dinâmica uterina e a dilatação cervical através do toque vaginal?

- É capaz de preencher corretamente o partograma e interpretá-lo? (Identifica suspeita de anormalidades no seguimento do trabalho de parto diante da interpretação do partograma.)

- Indica corretamente e interpreta a cardiotocografia?

- Diferencia o parto eutócico do parto distócico?
- É capaz de realizar um parto eutócico fazendo todas as manobras necessárias, comunicando-se com a equipe de pediatria e demais profissionais de saúde assistentes?

- É capaz de fornecer atendimento ao puerpério imediato? (Faz a dequitação placentária corretamente, revisão de trajeto e períneo, contenção de pequenas hemorragias identificando possíveis anormalidades.)

- Reconhece situação de urgência/emergência sendo capaz de comunicar-se prontamente com a equipe para solicitar apoio? (Faz o manejo da equipe delegando atribuições para resolução rápida de intercorrências.) 


\section{Quadro 3. APC 9 - PRINCÍPIOS DA CIRURGIA GINECOLÓGICA E ATENDIMENTO PERIOPERATÓRIO.}

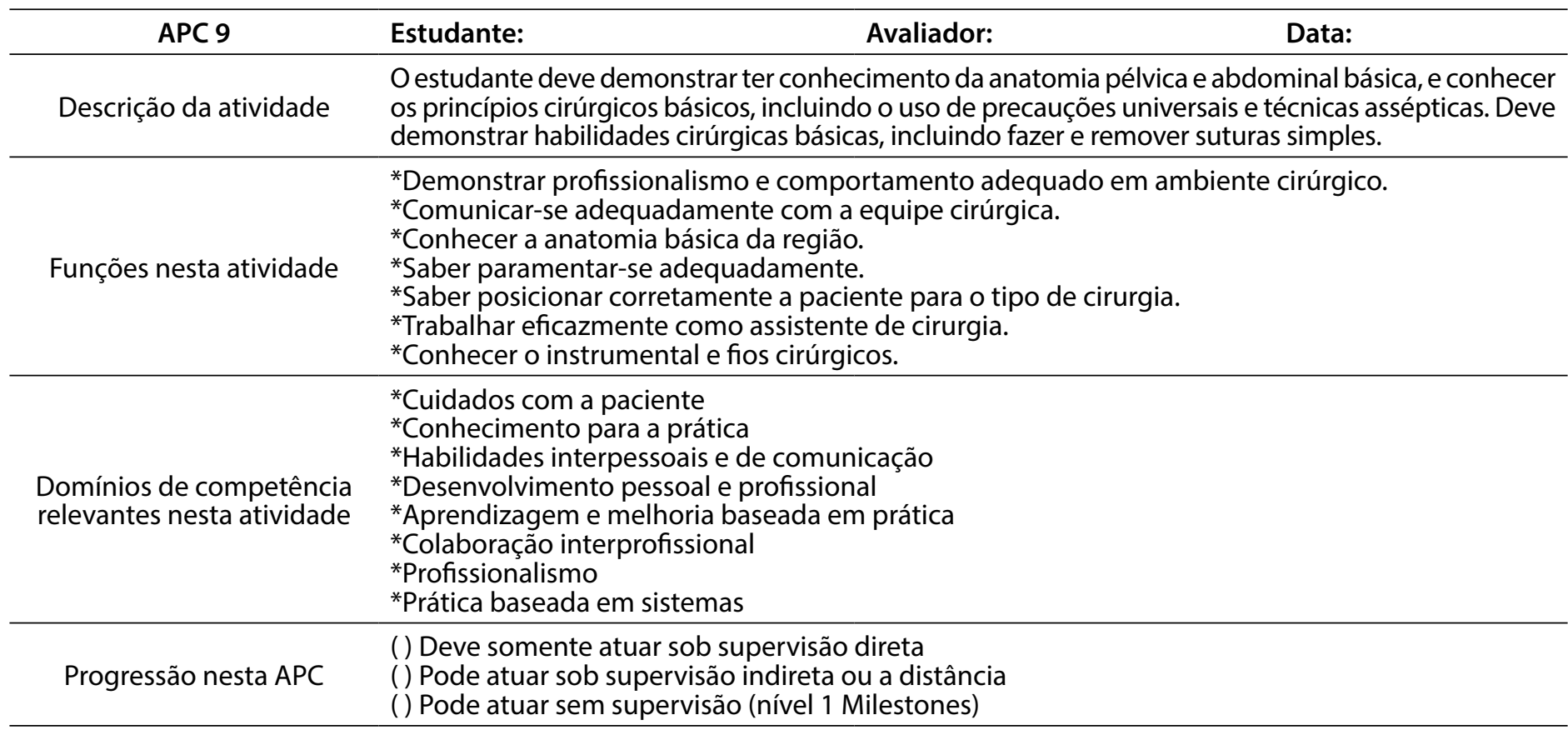

\section{LV - APC 9: princípios da cirurgia ginecológica e atendimento perioperatório}

Quando o estudante irá participar de cirurgia ginecológica:

- O estudante demonstra profissionalismo e comportamento adequado ao ambiente cirúrgico?

- O estudante realiza os passos básicos de lavagem das mãos e sabe paramentar-se corretamente?

- Conhece as precauções universais e técnicas de antissepsia, assepsia e esterilização?

- É capaz de comunicar-se adequadamente com a equipe cirúrgica e auxiliares?

- Demonstra conhecer a anatomia pélvica e abdominal básica, nomeando corretamente as estruturas quando solicitado?

- Sabe posicionar corretamente a paciente na mesa cirúrgica de acordo com o procedimento a ser realizado?

- Conhece o instrumental básico de cirurgia e os tipos de fios?

- O estudante é capaz de realizar e remover suturas simples?

\section{DISCUSSÃO}

O egresso do curso de medicina deve estar no correspondente ao nível 1 do Projeto Pilares, demonstrando deter o conhecimento mínimo exigido para atuação na área de ginecologia e obstetrícia. É esperado portanto que, no primeiro dia de egresso da faculdade de medicina, o profissional possa estar apto a exercer os requisitos mínimos e necessários em ginecologia e obstetrícia que propiciam o adequado atendimento à mulher e à gestante. Então, esse profissional deverá ser capaz de executar esses requisitos mínimos sem supervisão.

As Diretrizes Curriculares Nacionais de 2014 para o curso de medicina vieram sedimentar o perfil desejado do egresso sobre as bases da formação geral, humanista, crítica, reflexiva e ética devendo capacitar o graduado a atuar nos diferentes níveis de atenção à saúde. Para tal, coloca a necessidade de articulação entre conhecimentos, habilidades e atitudes para o futuro exercício profissional ${ }^{16}$. As APCs podem atender a este contexto também durante a graduação uma vez que possibilitam tanto ensinar quanto averiguar se o estudante é competente para desempenhar determinada tarefa que exija dele demonstrar integração entre o conhecimento, habilidade e atitude necessários para tal.

Grupos em outras especialidades médicas e da enfermagem no Brasil estudam o uso das APCs na graduação. Neste trabalho, trazemos o ineditismo das APCs com listas de verificação para o ensino e avaliação das competências adquiridas na área de ginecologia e obstetrícia durante a graduação médica.

Quando o avaliador assinala as duas primeiras opções no campo "Progressão nesta APC", ao estudante ainda não pode ser "confiada" determinada tarefa para que ele a execute sem supervisão. Isso demanda a necessidade de repetição dessa APC quantas vezes forem necessárias até que o estudante receba a avaliação de que "Pode atuar sem supervisão". Quando o estudante atingiu o grau de progressão no desempenho da APC, o avaliador o considera "confiável" para realizar as tarefas 
descritas e isso é o que se espera do estudante ao final da graduação. Assim também é o esperado no primeiro dia da residência médica, ou seja, o egresso alinha-se ao nível 1 do "Projeto Pilares para Obstetrícia e Ginecologia".

O modelo das APCs assim aplicado demonstra que o estudante é capaz de realizar tarefas sem supervisão quando chegar ao final da graduação, que é o que se espera dele ao formar-se médico. O estudante pode repetir a APC quantas vezes forem necessárias durante o curso de medicina, pode observar o seu progresso no aprendizado das competências propostas naquela APC e corrigir as falhas e desvios ainda sob supervisão. Isso proporciona a ele maior habilidade técnica e destreza na realização de procedimentos práticos, além de desenvolver posturas e atitudes na solução de cada tema proposto. Essas possibilidades, não obstante, enfrentam críticas relacionadas à efetividade e viabilidade da educação médica baseada em competências ${ }^{14,15}$. O modelo das APCs possibilita o aprendizado das competências na medida em que facilita observar os pontos fracos na formação médica e corrigi-los antes de o futuro profissional atingir o mercado de trabalho ou a residência médica. Também proporciona ao próprio estudante a visualização do que precisa ser aprimorado no seu aprendizado.

A lista de verificação com descrição minuciosa do que é necessário alcançar na realização de cada tarefa pode ser preenchida com base na observação e acompanhamento de uma pessoa (residente de ginecologia e obstetrícia ou um monitor) com mais conhecimento na área do que o estudante que realiza a APC; não implicando - assim - aumento de gastos para sua implantação e a necessidade de contratação de vários docentes para esse fim. No entanto, o estudante também deve passar pela avaliação final de um docente; importante que o estudante seja avaliado por docentes da área pelo menos algumas vezes no decorrer da graduação.

Construir um currículo prático usando APCs pode ser entendido como uma "Estratégia instrucional baseada em tarefas". Seguindo cinco princípios de instrução: foco na tarefa, ativação, demonstração, aplicação, integração ${ }^{13}$. Para fins educacionais, não é suficiente identificar as APCs apenas como uma simples lista de tarefas ou títulos; a maioria das formulações de tarefas está aberta a múltiplas interpretações. Para permitir uma decisão de atribuição, deve haver especificações; para ilustrar isso, se a APC for "Realizar anamnese com foco em ginecologia e obstetrícia" (APC 1), a decisão de atribuição deve incluir especificações e limitações. Por exemplo, para estudantes de medicina, pacientes de alto risco que precisam de cuidados urgentes são inadequados. Da mesma forma, o estudante do último ano pode examinar e avaliar uma paciente com condição conhecida, fazer exames diagnósticos complementares (APC 7) e conduzir o trabalho necessário a ser feito que deverá ser finalizado com a supervisão de alguém do corpo clínico. Além disso, a abordagem matricial para o uso de APCs requer a especificação de quais competências devem estar presentes antes que os participantes possam ser confiáveis para agir sem supervisão ou com supervisão apenas indireta.

É necessário um plano de avaliação para orientar os formandos na sua preparação para decisões de atribuição ${ }^{13}$.

As APCs podem ser vantajosas para a graduação. Estas centram-se na continuidade e progressão do desenvolvimento dos estudantes, na generalização e aplicabilidade através da continuidade dos princípios subjacentes às APCs, e no reconhecimento e garantia de qualidade do trabalho dos estudantes no local de trabalho clínico ${ }^{8}$. O fim da escola médica pode ser visto como a conclusão de um período de desenvolvimento específico com suas próprias expectativas de competência.

É preciso demonstrar ao público e à comunidade acadêmica que a educação médica baseada em competências está centrada em uma sólida teoria educacional e contribui não só para a formação profissional de médicos que incorporam os hábitos de trabalho para melhorar o atendimento ao paciente e à população, mas também contribui com os sistemas de assistência ${ }^{17}$.

\section{CONCLUSÃO}

A utilização das APCs durante a graduação vem agregar valor à educação médica baseada em competências, pois facilita e operacionaliza o aprendizado, o desenvolvimento e a observação da aquisição dos conhecimentos, habilidades e atitudes exigidos na execução de cada tema em ginecologia e obstetrícia, permitindo - assim - a formação de um profissional competente. Esse estudo inovador para o ensino da ginecologia e obstetrícia na graduação médica traz a descrição pormenorizada da tarefa a ser cumprida, assim como a lista de verificação para conferência dos itens realizados pelo estudante, proporcionando ao avaliador clareza quanto ao que deve ser executado e avaliado, de forma prática e rápida, não onerando em tempo ou gastos extras as instituições de ensino com a utilização das APCs. Além disso, as APCs e suas respectivas listas de verificação - estando sempre à disposição do estudante durante a graduação - possibilitam a oportunidade de serem repetidas quantas vezes necessárias para o aprendizado e domínio das funções solicitadas, as quais o estudante visualiza previamente na lista de verificação. Isso permite ao estudante uma autoavaliação ao identificar a necessidade de treinamento e aprofundamento em determinado assunto ou correção de falhas e desvios de aprendizado, sendo uma das maiores vantagens na utilização das APCs durante a graduação. 
Outra vantagem é que uma mesma APC pode ser inserida em vários momentos no decorrer da graduação médica para o aprendizado da ginecologia e obstetrícia. Algumas APCs são inseridas no começo do curso e permanem até o final; outras são inseridas em anos mais avançados, conforme o aprofundamento esperado. Essa "maleabilidade" na utilização deste modelo proporciona a facilidade de sua inserção tanto no ensino médico nos moldes tradicionais como naquele com metodologias ativas.

Ao final do curso de medicina, a capacidade de realização de todas as APCs sugeridas ao longo da graduação identifica um egresso competente em ginecologia e obstetrícia naquilo que concerne ao médico generalista. No entanto, necessitamos realizar mais estudos a fim de avaliar a aplicabilidade das APCs na graduação, o que já vem sendo realizado em um projeto piloto que nos trará dados futuros.

\section{CONTRIBUIÇÃO DOS AUTORES}

Os autores declaram que contribuíram igualmente para a realização do trabalho.

\section{CONFLITO DE INTERESSES}

Declaramos não haver conflito de interesses.

\section{FINANCIAMENTO}

Declaramos não haver financiamento.

\section{REFERÊNCIAS}

1. Ten Cate O. Guia atualizado sobre Atividades Profissionais Confiáveis (APCs). Rev Bras Educ Med. 2019;43(1 Supl.1):721-30.

2. Aguiar AC, Ribeiro EC. Conceito e avaliação de habilidades e competência na educação médica: percepções atuais dos especialistas. Rev Bras Educ Med. 2010;34(3):371-8.

3. Passini Júnior R. Ensino da cirurgia ginecológica nos programas de residência médica do Brasil. Rev Bras Ginecol Obstet. 2007;29(2):61-6.
4. Ten Cate O. Nuts and bolts of entrustable professional activities. J Grad Med Educ. 2013;5(1):157-8.

5. ten Cate $\mathrm{O}$. Entrustability of professional activities and competency-based training. Med Educ. 2005;39(12):1176-7.

6. ten Cate O, Scheele F. Viewpoint: Competency-based postgraduate training: can we bridge the gap between theory and clinical practice? Acad Med. 2007;82(6):542-7.

7. Mulder $\mathrm{H}$, ten Cate $\mathrm{O}$, Daalder R, Berkvens J. Building a competency-based workplace curriculum around entrustable professional activities: the case of physician assistant training. Med Teach. 2010;32:453-9.

8. Chen HC, O'Sullivan P, Teherani A, Fogh S, Kobashi B, Ten Cate O. Sequencing learning experiences to engage different level learners in the workplace: an interview study with excellent clinical teachers. Med Teach. 2015;37(12):1090-7.

9. Pangaro L, Ten Cate O. Frameworks for learner assessment in medicine. AMEE guide n78. Med Teach. 2013;35:1197-210.

10. Gentile P, Bencini R. Construindo competências: entrevista com Philippe Perrenoud, Universidade de Genebra. Nova Escola. 2000. [access in 7 mar 2017]. Available from: http://www.unige.ch/fapse/SSE/teachers/ perrenoud/php_main/php_2000/2000_3 1.html.

11. Association of American Medical Colleges. Core Entrustable Professional Activities for Entering Residency: Curriculum Developer's Guide 2014. [access in 18 jan 2017]. Available from: https://store.aamc.org/ downloadable/download/sample/sample_id/63/.

12. The Obstetrics and Gynecology Milestone Project. J Grad Med Educ. 2014;6(1 suppl):129-43. [access in $21 \mathrm{dec} 2016$ ]. Available from: https://doi.org/10.4300/JGME-06-01s1-07.

13. ten Cate O, Chen HC, Hoff RG, Peters H, Bok H, Van Der Schaaf M. Curriculum development for the workplace using entrustable professional activities (EPAs). AMEE Guide n99. Med Teach. 2015:1-20.

14. Talbot M. Monkey see, monkey do: a critique of the competency model in graduate medical education. Med Educ. 2004;38(6):587-92.

15. Whitehead CR, Kuper A. Faith-based medical education. Adv in Health Sci Educ. 2017;22(1):1-3.

16. Brasil. Ministério da Educação. Conselho Nacional de Educação. Resolução CNE/CES no 3, de 20 de jun. de 2014. Diretrizes Curriculares Nacionais do Curso de Graduação em Medicina e dá outras providências. Diário Oficial da República Federativa do Brasil, Brasília, 23 jun. de 2014; (Seção 1):8-11.

17. Carracio CL, Englander R, Van Melle E, Ten Cate O, Lockyer J, Chan MK, et al. Advancing competency-based medical education: a charter for clinicianeducators. Acad Med. 2016;91(5):645-9. 